\title{
Electronic state modification in laser deposited amorphous carbon films by the inclusion of nitrogen
}

\author{
Y. Miyajima, ${ }^{1, a)}$ G. Adamopoulos, ${ }^{1}$ S. J. Henley, ${ }^{1} \mathrm{~V}$. Stolojan, ${ }^{1}$ Y. Tison, ${ }^{1}$ E. Garcia-Caurel, ${ }^{2}$ \\ B. Drévillon, ${ }^{2}$ J. M. Shannon, ${ }^{1}$ and S. R. P. Silva ${ }^{1, \dot{b})}$ \\ ${ }^{1}$ Nano-Electronic Centre, Advanced Technology Institute, University of Surrey, Guildford, GU2 7XH, \\ United Kingdom \\ ${ }^{2}$ Laboratoire de Physique des Interfaces et des Couches Minces, UMR 7647 CNRS, Ecole Polytechnique, \\ 91128 Palaiseau Cedex, France
}

(Received 18 February 2008; accepted 10 July 2008; published online 17 September 2008)

\begin{abstract}
In this study, we investigate the effect of the inclusion of nitrogen in amorphous carbon thin films deposited by pulsed laser deposition, which results in stress induced modifications to the band structure and the concomitant changes to the electronic transport properties. The microstructural changes due to nitrogen incorporation were examined using electron energy-loss spectroscopy and Raman scattering. The band structure was investigated using spectroscopic ellipsometry data in the range of $1.5-5 \mathrm{eV}$, which was fitted to the Tauc Lorentz model parametrization and optical transmittance measurements. The dielectric constant evaluated using optical techniques was compared to that obtained with electrical measurements, assuming a Poole-Frenkel type conduction process based on the best fits to data. The electrical conduction mechanism is discussed for both low and high electric fields, in the context of the shape of the band density of states. By relating a wide range of measurement techniques, a detailed relationship between the microstructure, and the optical and the electrical structures of $a$ - $\mathrm{CN}_{x}$ films is obtained. From these measurements, it was found that, primarily, the change in density of the film, with increasing nitrogen pressure, affects the band structure of the amorphous carbon nitride. This is due to the fact that the density affects the stress in the film, which also impacts the localized states in the band gap. These results are supported by density of states measurements using scanning tunneling spectroscopy. (C) 2008 American Institute of Physics. [DOI: 10.1063/1.2977718]
\end{abstract}

\section{INTRODUCTION}

During the last few years, carbon nitride and its counterpart, amorphous carbon nitride $\left(a-\mathrm{CN}_{x}\right)$, has emerged as a material of high technological and scientific interest. ${ }^{1-6}$ The interest in carbon nitride has been renewed due to its potential as an electronic thin film for both cold cathode displays ${ }^{7}$ and electrode materials in electrochemical studies of water treatment. ${ }^{8}$ Electronic properties have been reported for both hydrogenated and nonhydrogenated amorphous carbon nitride. In particular, it is found that nitrogen incorporation has a beneficial effect on electric field emission at low microscopically applied fields by reducing the threshold electric field. ${ }^{9,10}$ The efficient inhibition of redox decomposition reactions of water using $a-\mathrm{CN}$ or $a-\mathrm{CN}: \mathrm{H}$ electrodes over a 3-4 $\mathrm{V}$ potential window, as compared to only about $2 \mathrm{~V}$ for metal electrodes, has also introduced many more potential applications. ${ }^{11,12}$ Additionally, the deposition of nitrogencontaining amorphous carbon has received particular attention following the theoretical predictions of an ultrahard silicon-nitride-like phase, i.e., $\beta-\mathrm{C}_{3} \mathrm{~N}_{4} \cdot{ }^{13,14}$ According to these predictions, this phase would have insulating properties, hardness, and thermal conductivity comparable to that of diamond.

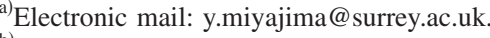

b) Author to whom correspondence should be addressed. Electronic mail: s.silva@surrey.ac.uk. FAX: + 441483686081.
}

Carbon nitride thin films can be deposited by a variety of deposition techniques, namely sputtering, ${ }^{15}$ plasma enhanced chemical vapor deposition, ${ }^{16,17}$ filtered cathodic vacuum arc (FVCA) ${ }^{18,19}$ electron cyclotron wave resonance (ECWR), ${ }^{20}$ mass selected ion beam deposition, ${ }^{21}$ and integrated distributed electron cyclotron resonance. ${ }^{22}$ Among the deposition techniques, pulsed laser deposition (PLD) (Refs. 23 and 24) is a well-established technique for $a$ - $\mathrm{CN}_{x}$ thin film deposition that allows for the tuning of the structural, optical, and electronic properties under varying growth conditions. Understanding the structure and the physics of amorphous carbon, as well as its alloys, is essential to improve electronic properties of carbon-based amorphous semiconductors such as carrier mobility, dielectric properties of low $k$ carbon-based films, ${ }^{25-27}$ as well as electron emission from carbon cold cathodes. $^{7}$

This study aims to investigate a series of PLD grown carbon nitride films with the aim of correlating the microstructure with the optical and the electronic transport properties for varying laser fluences and $\mathrm{N}_{2}$ pressure.

\section{EXPERIMENTAL DETAILS}

\section{A. Film deposition}

PLD of nonhydrogenated carbon nitride films have been investigated quite extensively over the past years. Most films have been produced by deposition onto a suitable substrate following nanosecond ultraviolet pulsed laser ablation (PLA) 
of a graphite target in a low-pressure nitrogen gas atmosphere. $^{28-30}$ The ablation apparatus used in the present study consisted of a high-vacuum stainless-steel chamber that was evacuated using a turbo molecular pump, backed by a mechanical pump, to a base pressure of $10^{-6}$ Torr. The chamber was back filled with $\mathrm{N}_{2}$ during thin film deposition. The incident laser beam was directed through one of the chamber side arms that was sealed with a quartz window and focused onto the target at $45^{\circ}$ to the surface. The target, a 10 $\mathrm{cm}$ in diameter disk of highly oriented pyrolytic graphite (Kurt J. Lesker, 99.99\% purity), was rotated to minimize repeated ablation of the same spot. A KrF Lambda Physik excimer laser (LPX 210i), operating at $248 \mathrm{~nm}$ with $25 \mathrm{~ns}$ full width at half-maximum (FWHM) pulse duration, was used as the UV pulsed laser source. The surface of the target was cleaned using laser ablation with a laser fluence of $4 \mathrm{~J} / \mathrm{cm}^{2}$ before depositions. The substrates were placed 6 $\mathrm{cm}$ from the graphite target. A series of $a-\mathrm{C}$ and $a-\mathrm{CN}_{x}$ films were deposited onto quartz, $c$-Si, and mica substrates at laser density power between 4 and $12 \mathrm{~J} / \mathrm{cm}^{2}$ for various nitrogen pressures with the repetition rate of $10 \mathrm{~Hz}$. Quartz and $c$-Si substrates were cleaned using a series of organic solvents, rinsed with de-ionized (DI) water, and dried using $\mathrm{N}_{2}$ gas. Deposition on mica was carried out immediately after cleaving. The typical thicknesses of the PLD deposited films were around $30 \mathrm{~nm}$.

\section{B. Electron energy loss spectroscopy}

The $s p^{2}$ content of our films was estimated using electron energy-loss spectroscopy (EELS) technique. ${ }^{19,31-33}$ The $s p^{2}$ fraction can be obtained from EELS using the peak corresponding to a transition from the occupied $1 s$ to empty $\pi^{*}$ states in the carbon $K$ edge spectra. The area of the peak was normalized within the region up to $294 \mathrm{eV}$ and the area of the peak was compared with graphite that is $100 \% s p^{2}$ bonded carbon. ${ }^{34}$ Additionally, the nitrogen content can be evaluated by comparing the carbon $K$ edge and a nitrogen $K$ edge. ${ }^{35}$ The valence electron density of the film can be evaluated from plasmon energy in the low loss spectrum. It must be noted that the density of the film can be obtained assuming that the number of valence electrons is four for carbon and five for nitrogen. ${ }^{36}$

A CM200 Philips Supertwin transmission electron microscope (TEM) operating at $200 \mathrm{kV}$ with a $\mathrm{LaB}_{6}$ filament was used for measurements with a Gatan Imaging Filter GIF2000 spectrometer for EELS. Films with a thickness of 30-70 nm were floated off from the mica substrate by immersing in DI water and then placed on copper TEM grids. The obtained EELS spectra and results derived for the films' density, $s p^{2}$ and nitrogen content for varying nitrogen pressures, and laser power densities are shown in Figs. 1 and 2, respectively.

\section{Raman spectroscopy}

Raman spectroscopy is one of the most popular and powerful techniques for the nondestructive analysis of amorphous carbon and its alloys that provides important structural information. $^{37-40}$ Unpolarized visible Raman spectra were

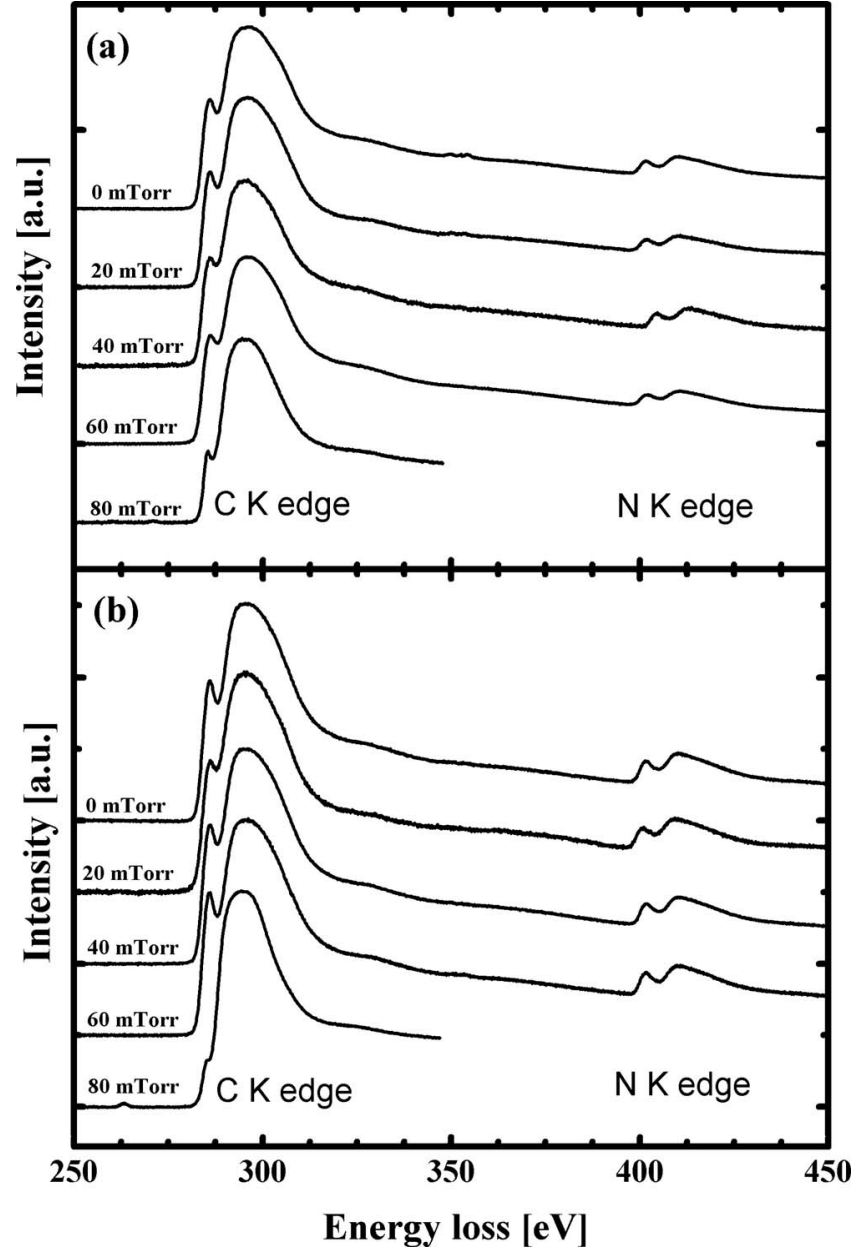

FIG. 1. The EELS spectra of $a-\mathrm{CN}_{x}$ films for different background nitrogen pressures and laser fluences of (a) 4 and (b) $12 \mathrm{~J} / \mathrm{cm}^{2}$.

obtained using the $514.5 \mathrm{~nm}$ line of an $\mathrm{Ar}^{+}$laser. A Renishaw micro Raman system 1000 spectrometer was used with a laser output of $15 \mathrm{~mW}$, which resulted in an incident power at the sample of about $1 \mathrm{~mW}$.

The recorded spectra between 800 and $1800 \mathrm{~cm}^{-1}$ are shown in Fig. 3. As a consequence of resonance effects, Raman is much more sensitive to $\pi$ bonds than to $\sigma$ bonds. In addition to the second order TO peak of the Si substrate at about $960 \mathrm{~cm}^{-1}$, the spectra show the usual amorphous carbon vibrational features, namely a $G$ peak at $1500-1580 \mathrm{~cm}^{-1}$ and a weaker $D$ peak around $1350 \mathrm{~cm}^{-1}$. The $G$ mode is the bond stretching mode between two $s p^{2}$-hybridized $C$ sites (olefinic or aromatic) while the $D$ mode is the breathing motion of $s p^{2}$-aromatic rings usually activated with disordered carbon where the graphene network has been disrupted.

The Raman spectra was curve fitted using the Breit-Wigner-Fano $^{41,42}$ line shape to the $G$ peak and Lorentzian line shape to the $D$ peak. ${ }^{37}$ The fitting results for the integrated $D$ and $G$ intensities ratio, i.e., the $G$ line width and the $G$ line position $\left[\omega_{\max }(\right.$ Ref. 37)], are shown in Fig. 4.

\section{Spectroscopic ellipsometry}

The $a$-C films were studied with ex situ UV visible spectroscopic ellipsometry (SE) in the range of photon energies 


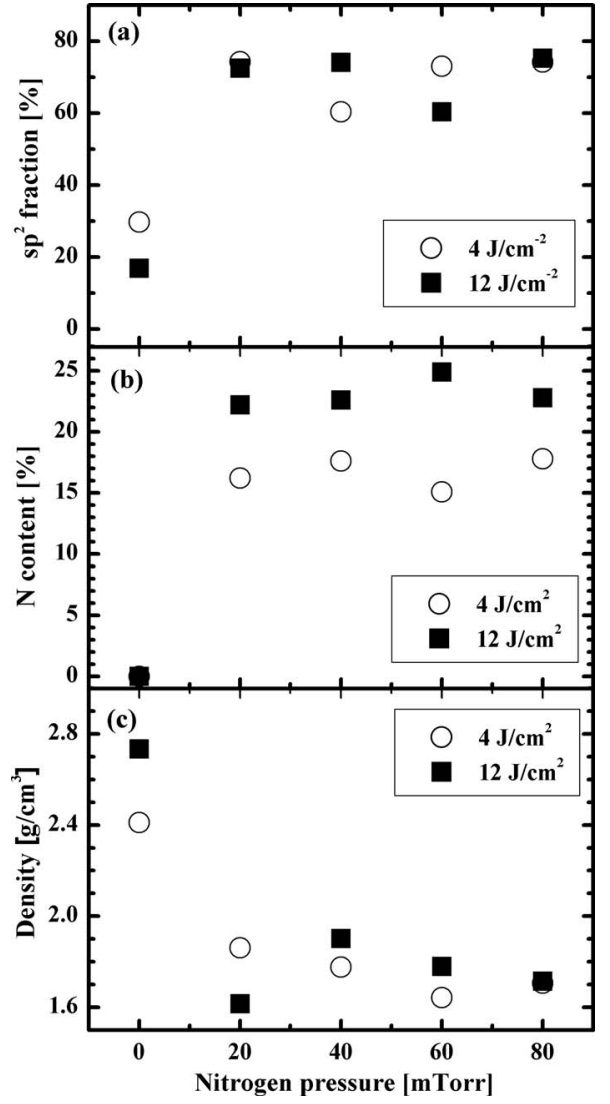

FIG. 2. The nitrogen gas pressure against (a) $s p^{2}$ fraction, (b) $\mathrm{N}$ content, and (c) density for laser fluences of 4 and $12 \mathrm{~J} / \mathrm{cm}^{2}$.

from 1.5 to $5 \mathrm{eV}$ using the UVISEL system by Jobin Yvon. For the analysis, a Tauc-Lorentz (TL) analytical expression for the dielectric function ${ }^{43,44}$ was assumed. The SE provides the complex refractive index and allows the energies $E_{g}$ (for

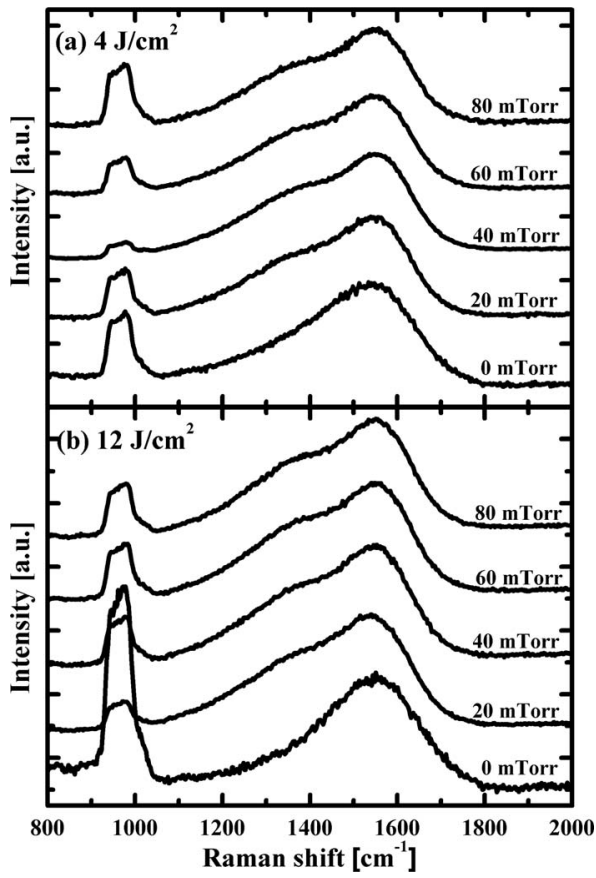

FIG. 3. The visible Raman spectra of $a$ - $\mathrm{CN}_{x}$ films for different nitrogen pressures and laser fluences of (a) 4 and (b) $12 \mathrm{~J} / \mathrm{cm}^{2}$.

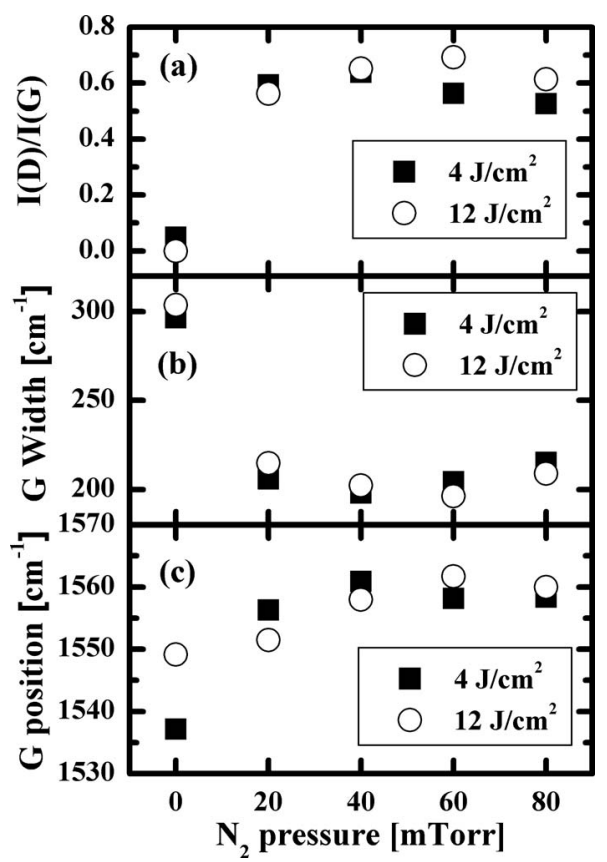

FIG. 4. (a) $I(D) / I(G)$ peak intensity ratio, (b) the FWHM of the $G$ peak, and (c) the $G$ peak position for laser fluences of 4 and $12 \mathrm{~J} / \mathrm{cm}^{2}$.

the onset of the optical transitions) and $E_{04}$ (where the absorption $\alpha=10^{4} \mathrm{~cm}^{-1}$ ) to be obtained. A four-parameter model $\left(A, C, E_{g}\right.$, and $\left.\varepsilon_{\text {inf }}\right)$ is sufficient to describe the optical functions of the thin film to the accuracy of the ellipsometer.

Jellison and Modine ${ }^{44}$ developed this model using the Tauc joint density of states (DOS) and the Lorentz oscillator. The imaginary part $\varepsilon_{i}$ of the dielectric function is given by the product of the imaginary part of Tauc's dielectric function with the Lorentz one. In the approximation of parabolic bands, Tauc's dielectric function describes interband transitions above the band edge. The new expression for $\varepsilon_{i}$ is set up as

$$
\begin{aligned}
& \varepsilon_{i}(E)=\left[\frac{A E_{o} C\left(E-E_{g}\right)^{2}}{\left(E^{2}-E_{o}^{2}\right)^{2}+C^{2} E^{2}} \cdot \frac{1}{E}\right] \text { for } E>E_{g}, \\
& \varepsilon_{i}(E)=0 \text { for } E \leq E_{g},
\end{aligned}
$$

where $E_{g}$ is the optical band gap, $A$ is the strength of the $\varepsilon_{i}$ peak, $C$ is the broadening term of the peak, and $E_{0}$ is the peak central energy; thus $E_{0}$ is always larger than $E_{g}$. The real part $\varepsilon_{r}$ of the dielectric function is derived from the expression of $\varepsilon_{i}$ using the Kramers-Kronig integration.

The TL expression is consistent with known physical phenomena within the limitations of the model. At large $E$, the $\varepsilon_{i}(E)$ of the TL model $\rightarrow 0$. This is consistent with observed behavior in the $\mathrm{x}$-ray and $\gamma$-ray regime, where it is known that the absorption coefficient is very small. Furthermore, $\varepsilon_{i}(E)=0$ below $E_{g}$. The only mechanisms that give a nonzero value of $\varepsilon_{i}(E)$ below the band gap are those that are explicitly ignored in the TL model, such as Urbach tail absorption and vibrational absorption in the infrared. Finally, the TL expression is consistent with the Kramers-Kronig derivation in that $\varepsilon_{r}(E)$ is determined by Kramers-Kronig 


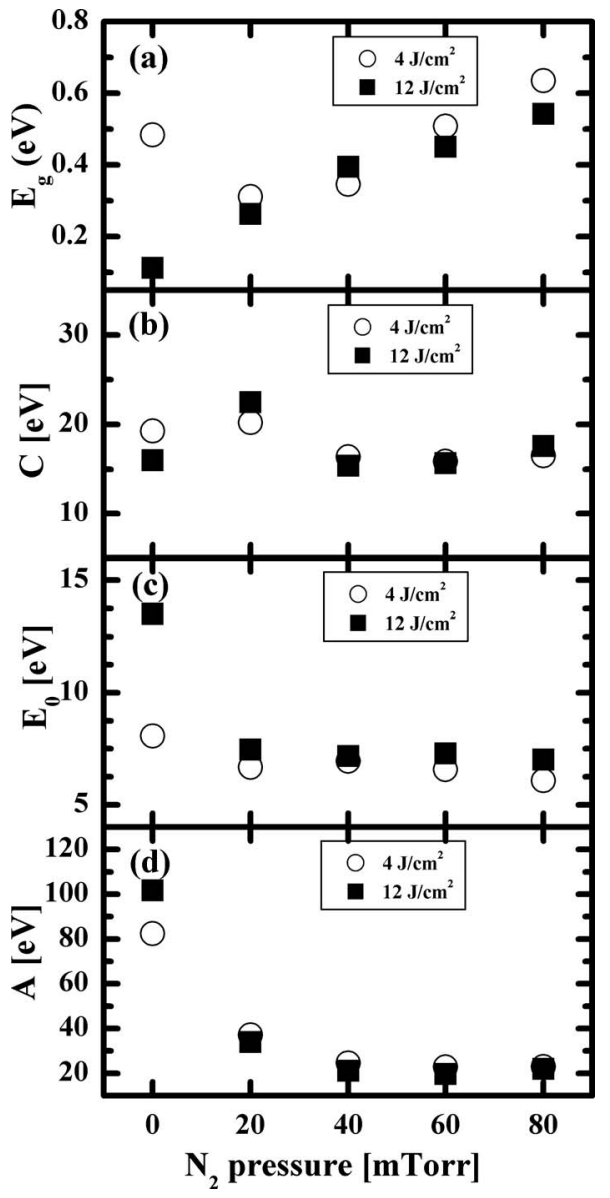

FIG. 5. SE TL model parameters. (a) Tauc optical band gap $E_{g}$, (b) the broadening factor $\mathrm{C},(\mathrm{c})$ the transition peak central energy $E_{0}$, and (d) the strength of the transition peak $A$.

integration. The high frequency dielectric constant $\left(\varepsilon_{\text {inf }}\right)$ is an additional fitting parameter that prevents $\varepsilon_{r}$ from converging to zero for energies below the band gap.

The fitted parameters of the TL model, as well as the refractive index $(n)$ and the extinction coefficient $(k)$ dispersions, are shown in Figs. 5 and 6, respectively. Here it should be pointed out that the model for inhomogeneous films, which is usually expressed by the volume fraction of void, was used.

\section{E. Ultraviolet-visible-near infrared optical transmittance}

A Cary 5000, Varian ultraviolet-visible-near infrared (UV-VIS-NIR) spectrometer was used for the optical transmittance measurements in the range between $190 \mathrm{~nm}$ $(\sim 6.5 \mathrm{eV})$ and $3000 \mathrm{~nm}(0.4 \mathrm{eV})$ in the dual beam mode. Films deposited on quartz substrates were used for these measurements.

The optical transmittance spectra is provided with complementary optical characteristics, namely, $E_{04}$ (corresponds to the absorption coefficient of $10^{4} \mathrm{~cm}^{-1}$ ) and the Tauc optical band gap. The optical characteristics of the films for various deposition conditions derived from SE and UVVIS-NIR are summarized in Fig. 7.

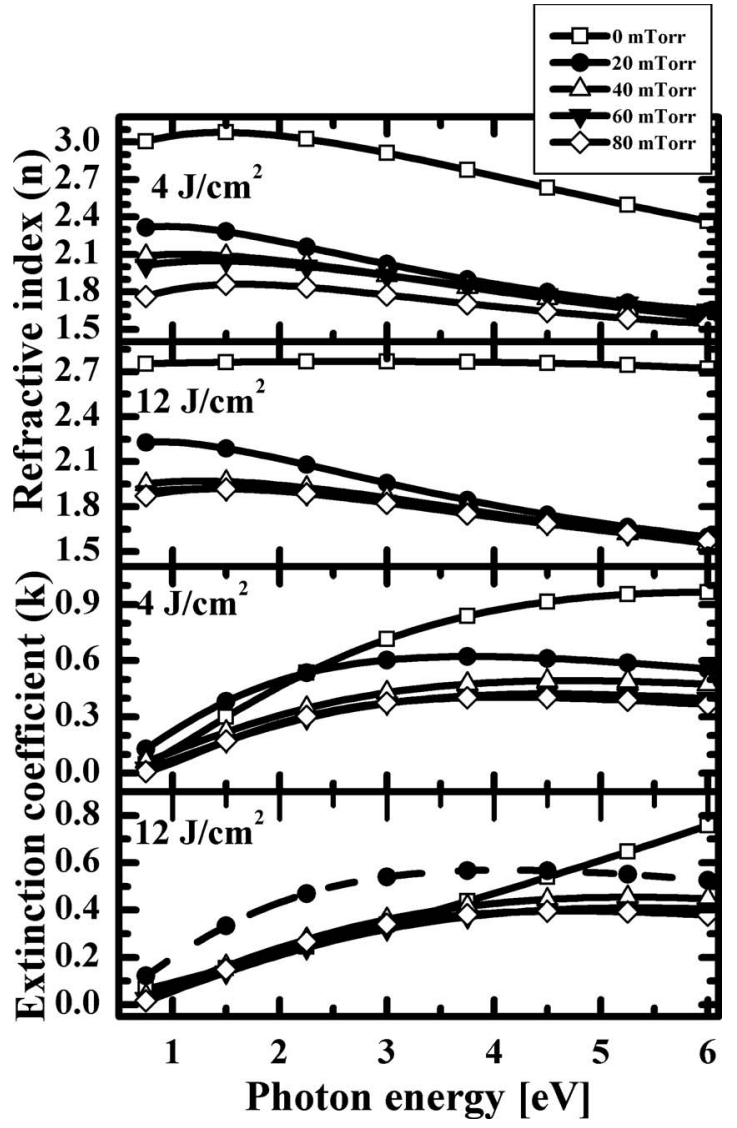

FIG. 6. $n$ and $k$ dispersions for different background nitrogen pressures and laser fluences of 4 and $12 \mathrm{~J} / \mathrm{cm}^{2}$.

\section{F. Electrical characterization}

Electrical characterization was carried out using sandwich structures. A highly doped $c$-Si wafer was used as a

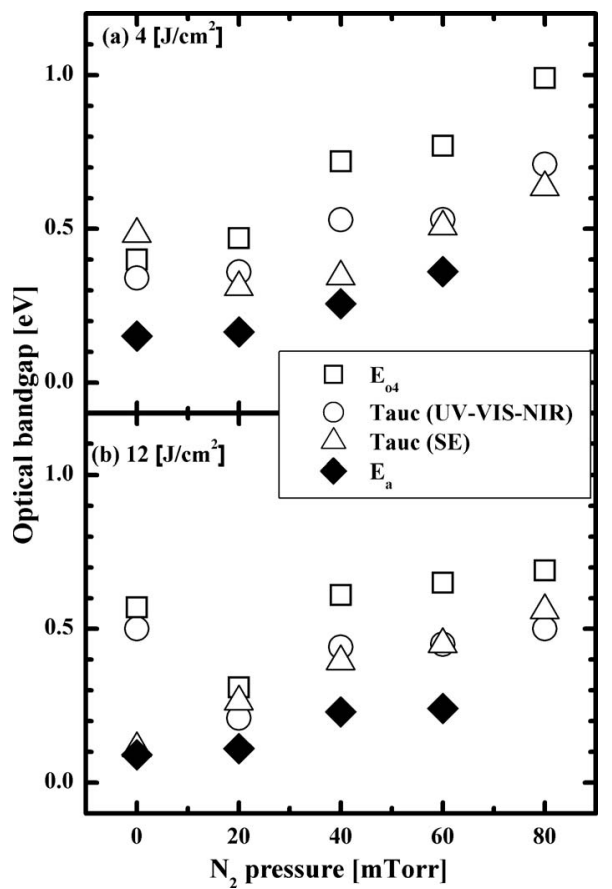

FIG. 7. The optical band gap ( $E_{04}$ and Tauc from UV-VIS-NIR and SE) and the activation energy measured at low electric fields against the nitrogen gas pressure for laser fluences of (a) 4 and (b) $12 \mathrm{~J} / \mathrm{cm}^{2}$. 


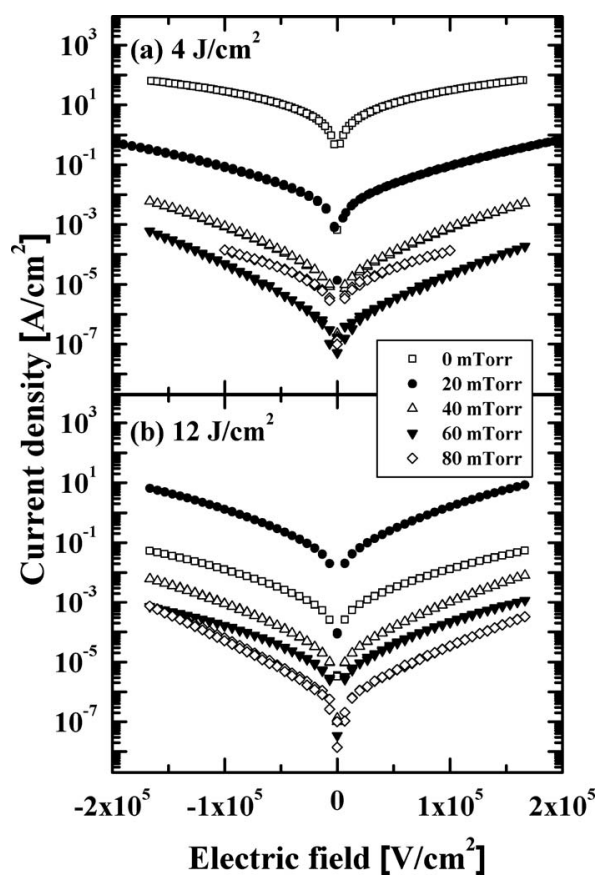

FIG. 8. J-E characteristics of devices deposited at various nitrogen pressures and laser fluences (a) 4 and (b) $12 \mathrm{~J} / \mathrm{cm}^{2}$.

substrate for the films and $100 \mathrm{~nm}$ of sputtered aluminum was used to form a back contact. A circular top electrode with an area of $1.5 \times 10^{-4} \mathrm{~mm}^{2}$ was fabricated using a shadow mask. The top electrodes were de sputtered and comprised of $50 \mathrm{~nm}$ of $\mathrm{Cr}$ coated with $50 \mathrm{~nm}$ of Au. The thickness of $a-\mathrm{CN}_{x}$ films was about $30 \mathrm{~nm}$.

Electrical measurements were carried out using a Keithley 236 source meter with a temperature controlled stage. The electrical characteristics at high electric fields were measured at room temperature. In order to evaluate the activation energy at low electric fields, the temperature was varied between room temperature and $80^{\circ} \mathrm{C}$. The Current Density-Electric Field characteristics are shown in Fig. 8. The activation energy $E_{a}$ is plotted out in Fig. 7, which was derived by assuming an Arrhenius relationship between current and inverse temperature.

\section{G. Scanning tunneling spectroscopy}

Scanning tunneling spectroscopy (STS) experiments were performed at room temperature in a commercial ultrahigh vacuum scanning tunneling microscope in the ultra high vacuum (UHV-STM) (Omicron VT multiscan STM) equipped with a scanning electron microscopic column using electrochemically etched tungsten tips. During the experiment, the base pressure in the UHV chamber was 2 $\times 10^{-11}$ mbar. The STS current-voltage curves were measured in the range of -1.5 to $+1.5 \mathrm{~V}$ for at least 16 positions on a $100 \times 100 \mathrm{~nm}^{2}$ image, and are averaged in order to obtain the DOS corresponding to a large area. Two samples were measured, and both $a-\mathrm{C}$ and $a-\mathrm{CN}_{x}$ films were deposited with the laser fluence of $4 \mathrm{~J} / \mathrm{cm}^{2}$.

\section{RESULTS AND DISCUSSION}

PLA of graphite results in the deposition of quite different carbonaceous structures depending on the pressure of the ambient gas in the chamber. The energy of the species involved with the growth can also be changed by applying an external bias to the chamber. A comparison of the PLA deposited material to that deposited using the conventional cathodic arc process can be found in the literature. ${ }^{45}$ Henley et $a l .{ }^{28}$ investigated the PLA mechanism of a graphite target in a chamber that contains Ar. The energy of the ablated carbon ions exceed $100 \mathrm{eV}$, the optimal energy for $s p^{3}$ bond formation, in the vacuum when a laser fluence of $10 \mathrm{~J} / \mathrm{cm}^{2}$ is used. When the Ar pressure is increased due to the additional collisions of the gas ions with its concomitant decrease in mean free path, the plume speed decreases. When the $\mathrm{Ar}$ background pressure exceeds 20 mTorr, a shockwavelike plume movement is observed and the speed reduces to around $8 \mathrm{~km} / \mathrm{s}, 400 \mathrm{~ns}$ after the ablation. The speed reduces to $2.6 \mathrm{~km} / \mathrm{s}$ after $800 \mathrm{~ns}$ with the Ar background pressure at 154 mTorr. Additionally, Fuge et al. ${ }^{46}$ investigated the plume dynamics during nanosecond and femtosecond ablations of graphite in nitrogen. These studies must be carefully considered in order to understand the microstructural differences initially revealed by EELS and Raman.

Following Fuge et al. ${ }^{46}$ a nitrogen background pressure between 20 and 80 mTorr was chosen. This is because the plume shows a shockwavelike propagation for pressures above 20 mTorr, meaning that the transport of the carbon atoms/clusters shows diffusive rather than ballistic character at this pressure range. The most important factor that controls the deposition process as well as the nitrogen incorporation (and consequently the films structure) is the number of the collisions that occur between the nitrogen molecules and the laser ablated carbon atoms/ions/clusters in the chamber once the nitrogen gas is introduced. The mean free path of the nitrogen gas at the pressure of 0.75 mTorr $(1 \mathrm{mbar})$ has been reported to be about $5 \mathrm{~cm}^{47}$ Taking into account that this distance is shorter than the distance between the substrate and the target (6 $\mathrm{cm}$ in our experimental setup), and the nitrogen pressure is much higher (20-80 mTorr), we can safely assume that at least one collision of the ablated carbon species with a nitrogen molecule is expected before the carbon atom/cluster reaches the substrate. In such a diffusive transport regime, the nitrogen is expected to be introduced into the film microstructure. It is clear then that the increase in the nitrogen pressure results in an increase in the multiple scattering and reduces the speed of the plume (or equivalently the carbon species). The small but notable decrease of the film's density for increased nitrogen pressure [Fig. 2(c)] can be attributed to the reduced energy of the carbon species incident on the substrate. Similar results have been reported for PLA of graphite in an Ar atmosphere. ${ }^{28}$ Figure 2(b) demonstrates that the nitrogen pressure seems to have almost no effect on the nitrogen content. It has been shown that the peak of the emission of the $\mathrm{CN}$ radicals appears at about 8 $\mathrm{mm}$ from the target at a nitrogen background pressure of 10 mTorr. ${ }^{46}$ Additionally, the mean free path of nitrogen at this pressure is estimated around this distance, assuming that the 
$\mathrm{CN}$ radicals are formed at the initial collisions. Higher laser fluences ablate more energetic carbon species. ${ }^{48}$ These species have a higher probability of reacting with nitrogen and forming $\mathrm{CN}$ radicals, resulting in higher nitrogen incorporation, as shown in Fig. 2(b). One of the reasons for an increase in the $s p^{2}$ content with increasing nitrogen pressure [Fig. 2(a)] is due to the decrease in the plume speed as a result of collisions between the carbon species and the nitrogen molecules. The pressure range is sufficient to let these carbon species experience diffusive transport before reaching the substrate, and the incident speed is not high enough to create high $s p^{3}$ fraction films. Furthermore, N, which prefers to bond in a planer $s p^{2}$ configuration, dictates bond formation in the plume, which then gets transported to the substrate.

The above trends are confirmed by the Raman spectra characteristics that are shown in Fig. 3 and the analysis that follows in Fig. 4. Indeed, the $G$ peak remains almost constant with increasing nitrogen pressure (i.e., the nitrogen content). This indicates that $\mathrm{N}$ addition has replaced the $\mathrm{C}=\mathrm{C}$ olefinic groups with aromatic groups. Figure 4(c) shows the $G$-line width variation as a function of the nitrogen pressure. Just to note that the $G$ peak width indicates the bond angle distortions in the excited configurations. It has been reported ${ }^{49}$ that the $G$-line width decreases with increasing N content, indicating loss of disorder of small clusters for higher $\mathrm{N}$ content. In the nitrogen pressure range of 20-80 mTorr, where the results in a nitrogen content is about 25 at $\%$ in our films, no $G$-line variation has been observed. The $I(D) / I(G)$ ratio [Fig. 4(a)] shows the same trend. A major effect of $\mathrm{N}$ incorporation is the increase in the clustering of the $s p^{2}$ phase, which is indicated by the $D$ peak. The $I(D) / I(G)$ ratio variation with increasing nitrogen content confirms that the fundamental dependence of $I(D) / I(G)$ is on $s p^{2}$ clustering.

The TL SE model fitted parameters are shown in Fig. 5. If the valence and the conduction bands are treated as single peaks, the peak position of $a-\mathrm{CN}_{x}$ should shift toward the Fermi level, compared with $a-\mathrm{C}$, since the $\sigma$ and $\sigma^{*}$ bands become smaller in comparison to the $\pi$ and $\pi^{*}$ bands, which become larger. This explanation can be supported by the EELS $K$ edge data (see Fig. 1), which can be treated as a modification to the shape of the conduction band. This explains the drop observed in the mean peak position $E_{0}$ and the peak strength $A$, and the constant value of the broadening factor $C$. What is remarkable is the small increase in the optical band gap considering the large increase in nitrogen background pressure.

Figure 7 shows the optical band gap (both Tauc and $E_{04}$ ) that was derived using different techniques. These results are different to those reported in Ref. 49 where nitrogen incorporation decreases the optical band gap due to an increase in the $s p^{2}$ content of the film and its consequences. Similar results were reported on $t a-\mathrm{CN}_{x}$ deposited using FVCA and ECWR. ${ }^{50}$ On the other hand, dc sputtered low $s p^{3}$ fraction $a-\mathrm{CN}_{x}$ shows an increase in optical band gap with increasing N/C ratio. ${ }^{50}$ However, in the latter cases, the optical band gaps are larger than those of the present study; thus there must be another mechanism that reduces the optical band gap in PLD $a-\mathrm{C}$ and $a-\mathrm{CN}_{x}$ films.
The increasing optical band gap may be attributed to the decreased defect density in the band gap since the films get less dense. The obvious difference in the optical properties of the nitrogen free films grown at different laser fluences is due to the fact that the higher laser fluence forms higher quantities of $s p^{3}$ bonded material. Higher $s p^{3}$ fraction films show higher $E_{0}$ and $A$ values for the nitrogen free $a$-C, especially those grown at $12 \mathrm{~J} / \mathrm{cm}^{2}$. The small optical band gap $a-C$ films with high $s p^{3}$ fraction and dense film properties have been reported elsewhere, ${ }^{51}$ and the reduced band gap is attributed to the high internal stress. The internal stress in the $a$-C films deposited at more than $12 \mathrm{~J} / \mathrm{cm}^{2}$ is considered to be high due to the films being able to delaminate easily, whereas $a-\mathrm{CN}_{x}$ films have good adhesion to the substrates. Thus, the high $s p^{3}$ fraction films have high internal stress that may create localized states in the band gap and thus contribute to a low optical band gap.

The $J-E$ characteristics are shown in Fig. 8. It appears that the films become more resistive with increasing nitrogen pressure for both laser fluences used. This is consistent with the SE analysis, which concluded a lower defect density with increasing nitrogen pressure, as well as the optical analysis, which showed an increased band gap that would increase the resistivity of the films. The free carrier concentration in a semiconductor has an inverse exponential relationship to the optical band gap. It was found that some samples were under stress and failed at high electric fields. In most cases, electric fields of less than $2.0 \times 10^{5} \mathrm{~V} / \mathrm{cm}$ should be applied for reproducible results for these films due to their high stress. In the case of $a-\mathrm{CN}_{x}$ deposited at $80 \mathrm{mTorr}$ and a laser fluence of $4 \mathrm{~J} / \mathrm{cm}^{2}$, the field was limited to $1.0 \times 10^{5} \mathrm{~V} / \mathrm{cm}$.

Figure 7 also show the activation energy $E_{a}$ at low electric fields, which has comparable values to those of the optical band gap evaluated using different techniques.

A possible conduction mechanism for such low electric fields was reported to be band tail hopping, ${ }^{52}$ via defects (band tail) above the Fermi level. The defect states are thought to lie above the Fermi level and the electrical activation energy indicates the energy between the Fermi level and the defects. If the Fermi level lies in the middle of the band gap, band tail hopping seems to occur at the bottom of the empty $\pi^{*}$ band. It is consistent with the similar values measured for both of $E_{a}$ and optical band gaps.

Figure 9(a) shows the dielectric constant that was obtained from SE. In general, there is a good correlation between the dielectric constant obtained from SE and that obtained from conductivity measurements. Such a correlation is found for $a-\mathrm{C}$ and $a-\mathrm{CN}_{x}$ grown at 20 mTorr of nitrogen gas (see Fig. 9).

The conductivity of $a-\mathrm{CN}_{x}$ appears to be independent of the laser fluence and it decreases with increasing nitrogen pressure. This may also be related to the decrease in the number of defects in the band gap due to the decrease in the internal stress. It is believed that the decrease in the defect states at the midband gap is reflected by the decrease in the activation energy at low electric fields. In this region, the conduction mechanism appears to be hopping within a defect 


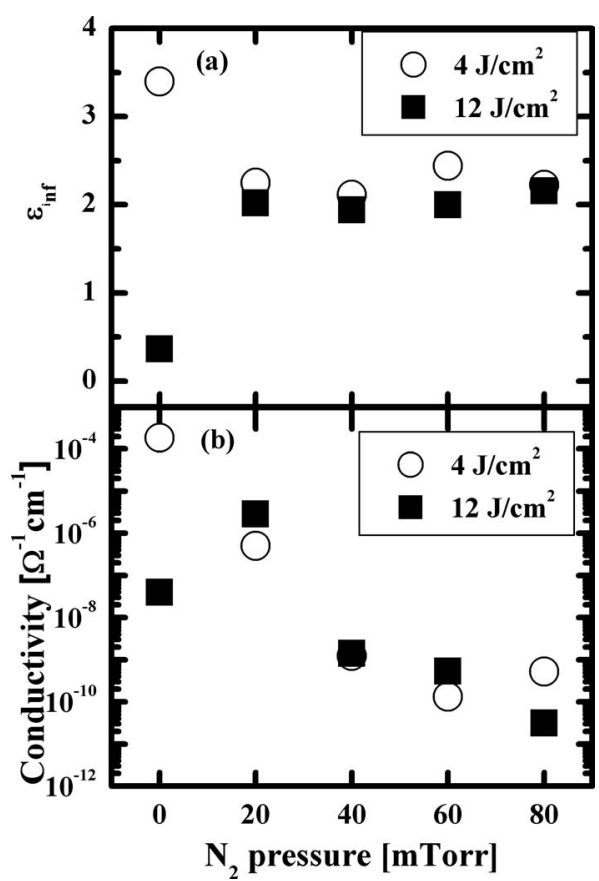

FIG. 9. The $\mathrm{N}_{2}$ pressure dependence of (a) the dielectric constant at high energy derived from SE and (b) the conductivity at low electric fields less than $10^{4} \mathrm{~V} / \mathrm{cm}$ at room temperature.

band, and the conductivity decreases with increasing activation energy and a decrease in the defect density within the band gap.

The conduction mechanism at high electric fields is normally different to that at low fields. ${ }^{53}$ Figure 10 shows the

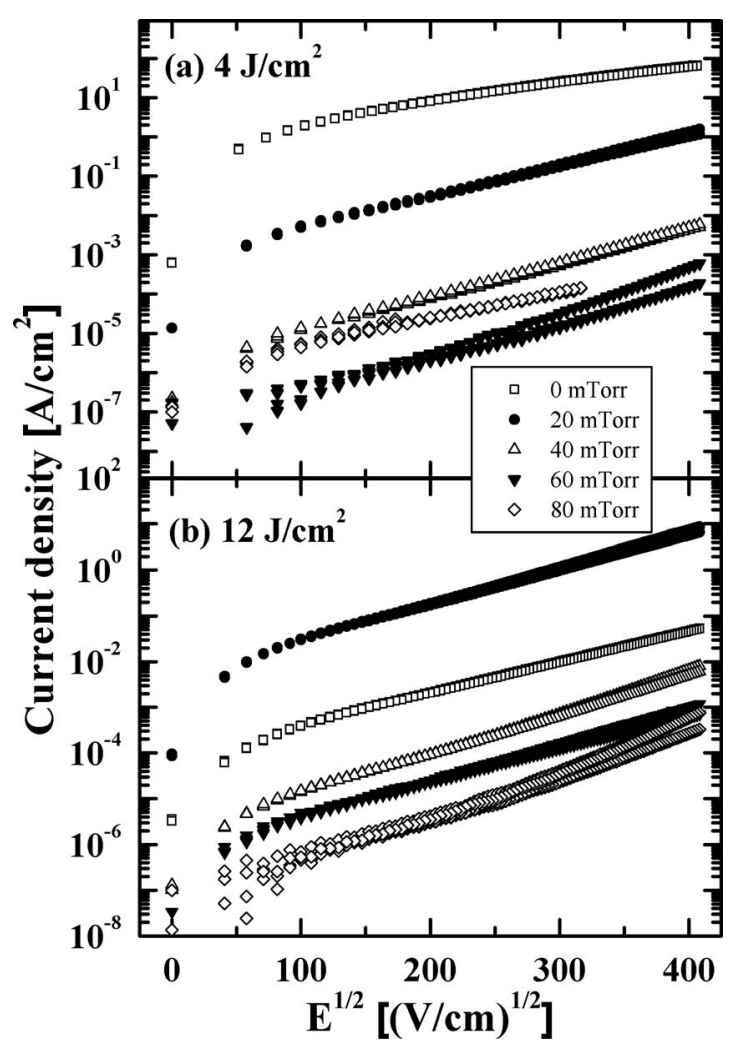

FIG. 10. PF plots against $\mathrm{N}_{2}$ pressure for laser fluences of (a) 4 and (b) $12 \mathrm{~J} / \mathrm{cm}^{2}$. $\log$ of the current density versus the square root of the electric field at room temperature as a function of the nitrogen pressure for laser fluences of 4 and $12 \mathrm{~J} / \mathrm{cm}^{2}$. At electric fields higher than $10^{4} \mathrm{~V} / \mathrm{cm}$, there is a linear dependence, revealing that the main conduction mechanism appears to be either Poole-Frenkel (PF) bulk limited or Schottky barrier contact limited conduction.

For these samples, two types of contacts were used. One was the $n^{+}$-Si substrate while the other was a $\mathrm{Cr}$ top contact. However, the $J-E$ characteristics seem to be symmetric, indicative that the conduction mechanism is not contact dominated. Thus, we come to the conclusion that the conduction mechanism for these films could be a bulk limited process such as PF conduction.

The current density $J$ due to PF conduction through the film is given by

$$
J=e N \mu^{*} E \exp \left\{\frac{-\left[\Phi-\left(\beta_{P F} / \gamma\right) \sqrt{E}\right]}{k T}\right\},
$$

where

$$
\beta_{P F}=\sqrt{\frac{e^{3}}{\pi \varepsilon_{r} \varepsilon_{0}}},
$$

$\Phi$ is the trap depth, $E$ the electric field across the film, $N$ the density of the neutral trapping centers, $\mu$ the effective mobility, and $\gamma$ is a coefficient that changes from one to two, depending on the number of the charged defects and the degree of compensation. When $\gamma=1$ is satisfied, the formula is similar to the classical PF conduction model. ${ }^{53}$ Here, it should be noted that the neutral trapping centers are not necessarily the same as the defects detected by electron spin resonance (ESR), which detects unpaired spins.

The application of the classical PF $(\gamma=1)$ conduction model of $a-\mathrm{C}$ and $a-\mathrm{CN}_{x}$ can provide us with an effective dielectric constant. These values, as well as those for the square of the refractive index $n$ (at $2 \mathrm{eV}$ ) assuming a low imaginary component, were derived from the TL model dispersion curves (Fig. 6) and are shown in Fig. 11 along with those calculated from single wavelength ellipsometry. It is demonstrated that the approximations that were used for both optical and electrical measurements are very consistent. Additionally, the decrease in the refractive index (at $2 \mathrm{eV}$ ) for increasing nitrogen gas pressure is consistent with the decrease in the films density, ${ }^{54}$ as discussed earlier.

If the PF conduction mechanism dominates at high fields, then the activation energy should be proportional to the square root of the electric field. A $a-\mathrm{CN}_{x}$ film that was grown at a laser fluence of $4 \mathrm{~J} / \mathrm{cm}^{2}$ in 20 mTorr nitrogen gas was measured to check for the change in activation energy with electric field, as shown in Fig. 12. The estimated trapping center depth was found to be about $0.16 \mathrm{eV}$. In addition, the product $\mathrm{N} \mu^{*}$ can also be evaluated using the above equation. For the device used in Fig. 12, $\mathrm{N} \mu^{*}$ was estimated to be about $3 \times 10^{15}(\mathrm{~cm} \mathrm{~V} \mathrm{~s})^{-1}$. Investigation using ESR provides a defect density of less than $10^{21} \mathrm{~cm}^{-3}$ for nonhydrogenated $a-\mathrm{C}$ (Ref. 55) and $10^{18} \mathrm{~cm}^{-3}$ for sputtered $a-\mathrm{CN}_{x}{ }^{56}$ Assuming that the density of the neutral trapping centers is less than this value, the mobility is higher than $10^{-4} \mathrm{~cm}^{2} / \mathrm{Vs}$ when 


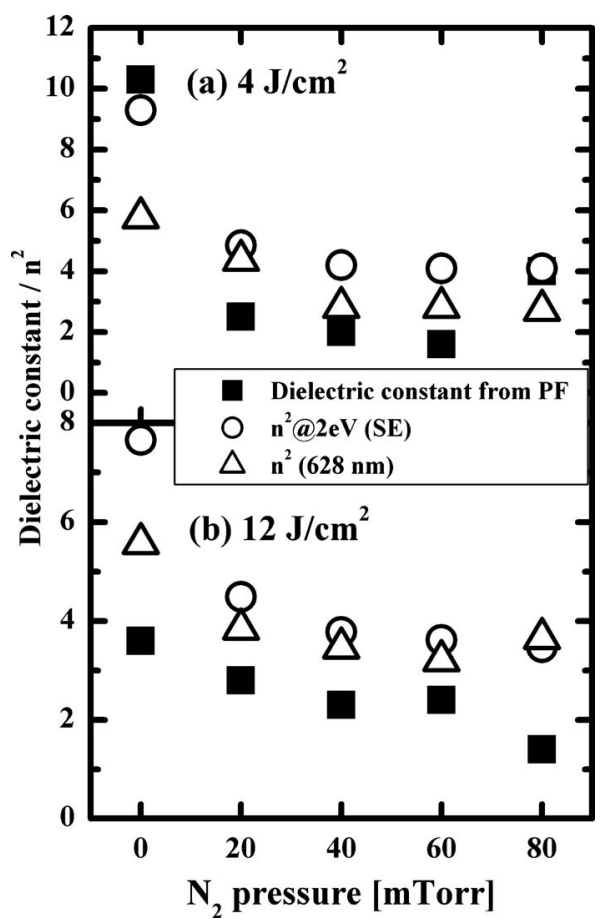

FIG. 11. The dielectric constant obtained from PF plots, and $n^{2}$ at $2 \mathrm{eV}$ extracted from $n$ and $k$ dispersions with monochromatic ellipsometry.

$\mathrm{N}<10^{18} \mathrm{~cm}^{-3}$ for $a-\mathrm{CN}_{x}$ films. This is consistent with the reported field effect mobility of $a-\mathrm{CN}_{x}$ being up to about $10^{-4} \mathrm{~cm}^{2} / \mathrm{V} \mathrm{s}^{57,58}$ The field effect mobility is estimated from low electric fields $\left(<10^{3} \mathrm{~V} / \mathrm{cm}\right)$ whereas the effective mobility in the conduction band used the PF plot at high electric fields $\left(>10^{4} \mathrm{~V} / \mathrm{cm}\right)$. At low electric fields, the conductive mechanism is thought to be a hopping process through the defect band, whereas the PF effect occurs at the bottom of the conduction band. Thus, the mobility estimated from PF plots can be higher than that extracted using field effect transistor measurements. It should also be pointed out that band tail hopping has been reported in $a-\mathrm{CN}_{x}$ films and that the PF effect might occur through defects in the band tail $^{22,52}$ instead of the edge of the conduction band.

Figure 13 shows the normalized conductance of $a-\mathrm{C}$ and $a-\mathrm{CN}_{x}$. The normalized conductance is proportional to the DOS, and a negative sample bias shows the valence band of the films, whereas a positive sample bias shows the conduction band of the films. From these DOS measured using STS

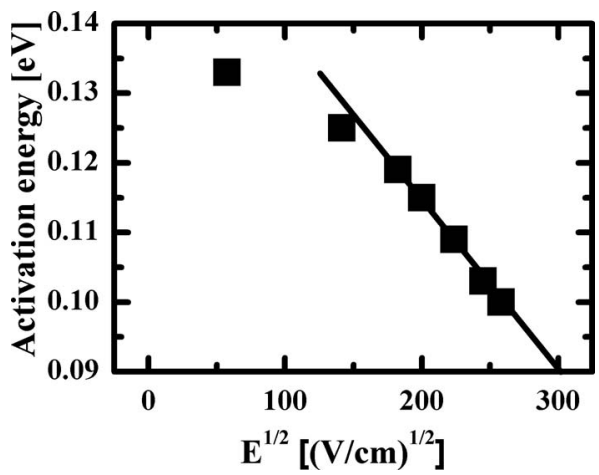

FIG. 12. The activation energy versus the square root of the electric field for $a$ - $\mathrm{CN}_{x}$ film deposited in $20 \mathrm{mTorr} \mathrm{N}_{2}$ gas with a laser fluence of $12 \mathrm{~J} / \mathrm{cm}^{2}$.

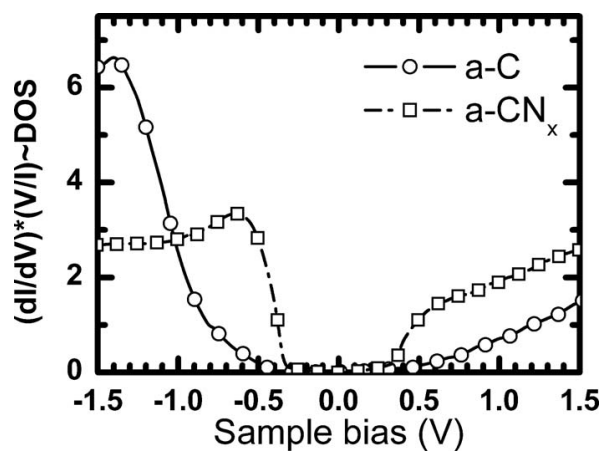

FIG. 13. The DOS derived from STS for PLD $a$-C and $a-\mathrm{CN}_{x}$ films.

under UHV conditions, the gap of $a-\mathrm{C}(\sim 0.9 \mathrm{eV})$ measured appears to be larger than that of $a-\mathrm{CN}_{x}(\sim 0.7 \mathrm{eV})$, which is in agreement with previous reports and literature with regards to $t a-\mathrm{CN}_{x}$ but in disagreement with those results reported for dc sputtered $a-\mathrm{CN}_{x}$. ${ }^{49,50}$ However, the optical band gap decreases when nitrogen is introduced. A remarkable feature of Fig. 13 is the shallow slope of the DOS in the conduction band. This is thought to be the reason why the optical band gap is smaller than that reported in previous reports. ${ }^{49,50}$ The long band tail narrows the optical band gap compared with the effective band gap for both $a$-C and $a-\mathrm{CN}_{x}$ films. This feature may be attributed to the high stress in these films.

Therefore, the band diagram of PLD $a-\mathrm{C}$ and $a-\mathrm{CN}_{x}$ can be illustrated, as shown in Fig. 14. The DOS of carbon films consist of $\pi$ and $\pi^{*}$ bands attributed to $s p^{2}$ hybridization, and $\sigma$ and $\sigma^{*}$ bands associated with $s p^{3}$ hybridization. Moreover, the band tail exists between $\pi$ and $\pi^{*}$ bands. The optical band gap attributed to the transitions from the occupied $\pi$ to the unoccupied $\pi^{*}$ bands, which are nonsymmetrical bands, was confirmed with the DOS obtained by STS analysis (see Fig. 7 and 12).

\section{CONCLUSION}

This study demonstrates the effect of nitrogen inclusion on the electronic states and the physical properties, including electrical properties, in $a$-C films deposited using PLD. The pressure range of nitrogen was chosen such that diffusive
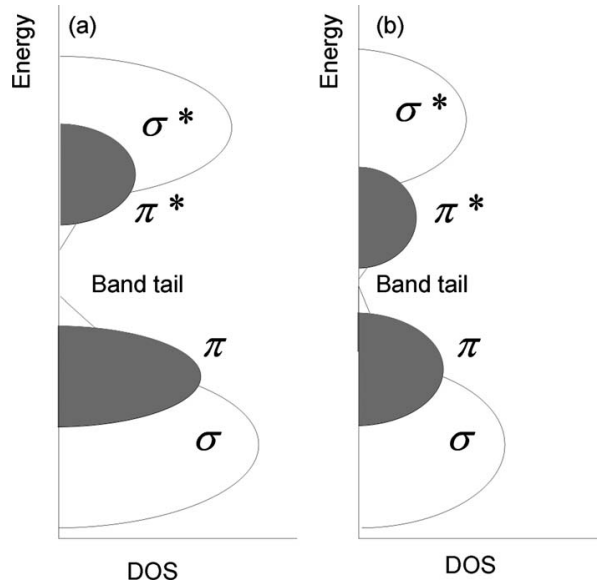

FIG. 14. The band diagram of PLD (a) $a-\mathrm{C}$ and (b) $a-\mathrm{CN}_{x}$. 
carbon transport occurred during deposition and incorporation of nitrogen into the films. A series of techniques were applied for structural, optical, and electrical characterization, namely, EELS, Raman spectroscopy, SE, and UV-VIS-NIR spectroscopy along with $I-V$ measurements. It has been shown that nitrogen incorporation changes the $s p^{3}$ fraction of the films. Once nitrogen was incorporated, no strong dependence between the nitrogen gas pressure, the laser fluence, and the microstructure was found. However, the background pressure during the deposition did affect the density, which can in turn affect the stress in the films, and hence the localized states in the band gap. Therefore, an increase in the nitrogen pressure decreases conductivity and increases the optical band gap. The presence of the long band tail is supported by the DOS close to the Fermi level obtained by STS measurements and is in agreement with the broadening factor $C$ obtained from SE. Thus, the presence of a long band tail should be carefully considered when using $a-C$ and $a-\mathrm{CN}_{x}$ films deposited by PLD in electronic devices.

Two types of conduction mechanism were found for low and high electric fields. Band tail hopping appears to be the dominant conduction mechanism at low electric fields while $\mathrm{PF}$ conduction was confirmed to dominate conduction at high fields. The fact that transport is dominated by hopping between localized neutral defects suggests that shallow nitrogen donors are not present in large concentrations.

\section{ACKNOWLEDGMENTS}

The authors are grateful to the EPSRC Swindon, U.K. for funding this program via a Portfolio Partnership award.

${ }^{1}$ M. Moseler, P. Gumbsch, C. Casiraghi, A. C. Ferrari, and J. Robertson, Science 309, 1545 (2005).

${ }^{2}$ S. Bhattacharyya, S. J. Henley, E. Mendoza, L. Gomez-Rojas, J. Allam, and S. R. P. Silva, Nat. Mater. 5, 19 (2006).

${ }^{3}$ Properties of Amorphous Carbon, edited by S. R. P. Silva (INSPEC, London, 2003).

${ }^{4}$ Amorphous Carbon: State of the Art, edited by S. R. P. Silva, J. Robertson, W. I. Milne, and G. A. J. Amaratunga (World Scientific, Singapore, 1997).

${ }^{5}$ J. Robertson, Mater. Sci. Eng., R. 37, 129 (2002)

${ }^{6}$ J. Windeln, C. Bram, H. L. Eckes, D. Hammel, J. Huth, J. Marien, H. Rohl, C. Schug, M. Wahl, and A. Wienss, Appl. Surf. Sci. 179, 167 (2001).

${ }^{7}$ S. R. P. Silva, J. D. Carey, G. Y. Chen, D. C. Cox, R. D. Forrest, C. H. P. Poa, R. C. Smith, Y. F. Tang, and J. M. Shannon, IEE Proc.: Circuits Devices Syst. 151, 489 (2004).

${ }^{8}$ H. Cachet, C. Debiemme-Chouvy, C. Deslouis, A. Lagrini, and V. Vivier, Surf. Interface Anal. 38, 719 (2006)

${ }^{9}$ G. A. J. Amaratunga and S. R. P. Silva, Appl. Phys. Lett. 68, 2529 (1996).

${ }^{10}$ B. S. Satyanarayana, A. Hart, W. I. Milne, and J. Robertson, Appl. Phys. Lett. 71, 1430 (1997).

${ }^{11}$ G. Adamopoulos, C. Godet, C. Deslouis, H. Cachet, A. Lagrini, and B. Saidani, Diamond Relat. Mater. 12, 613 (2003).

${ }^{12}$ K. S. Yoo, B. Miller, R. Kalish, and X. Shi, Electrochem. Solid-State Lett. 2, 233 (1999)

${ }^{13}$ A. Y. Liu and M. L. Cohen, Science 245, 841 (1989).

${ }^{14}$ A. Y. Liu, R. M. Wentzcovitch, and M. L. Cohen, Phys. Rev. B 39, 1760 (1989).

${ }^{15}$ R. Ohr, B. Jacoby, M. von Gradowski, C. Schug, and H. Hilgers, Surf. Coat. Technol. 173, 111 (2003).

${ }^{16}$ S. R. P. Silva and G. A. J. Amaratunga, Thin Solid Films 253, 146 (1994).

${ }^{17}$ S. R. P. Silva, B. Rafferty, G. A. J. Amaratunga, J. Schwan, D. F. Franceschini, and L. M. Brown, Diamond Relat. Mater. 5, 401 (1996).

${ }^{18}$ G. A. J. Amaratunga, V. S. Veerasamy, W. I. Milne, C. A. Davis, S. R. P. Silva, and H. S. Mackenzie, Appl. Phys. Lett. 63, 370 (1993).
${ }^{19}$ P. J. Fallon, V. S. Veerasamy, C. A. Davis, J. Robertson, G. A. J. Amaratunga, W. I. Milne, and J. Koskinen, Phys. Rev. B 48, 4777 (1993).

${ }^{20}$ S. Rodil, N. A. Morrison, W. I. Milne, J. Robertson, V. Stolojan, and D. N. Jayawardane, Diamond Relat. Mater. 9, 524 (2000).

${ }^{21}$ Y. Lifshitz, S. R. Kasi, and J. W. Rabalais, Phys. Rev. Lett. 62, 1290 (1989).

${ }^{22}$ C. Godet, N. M. J. Conway, J. E. Bouree, K. Bouamra, A. Grosman, and C. Ortega, J. Appl. Phys. 91, 4154 (2002).

${ }^{23}$ M. P. Siegal, J. C. Barbour, P. N. Provencio, D. R. Tallant, and T. A. Friedmann, Appl. Phys. Lett. 73, 759 (1998).

${ }^{24}$ S. R. P. Silva, S. Xu, B. K. Tay, H. S. Tan, H. J. Scheibe, M. Chhowalla, and W. I. Milne, Thin Solid Films 290-291, 317 (1996).

${ }^{25}$ A. Grill, Diamond Relat. Mater. 10, 234 (2001).

${ }^{26}$ M. Aono and S. Nitta, Diamond Relat. Mater. 11, 1219 (2002).

${ }^{27}$ M. Aono, S. Nitta, T. Katsuno, T. Itoh, and S. Nonomura, Appl. Surf. Sci. 159-160, 341 (2000).

${ }^{28}$ S. J. Henley, J. D. Carey, S. R. P. Silva, G. M. Fuge, M. N. R. Ashfold, and D. Anglos, Phys. Rev. B 72, 205413 (2005).

${ }^{29}$ A. A. Voevodin, J. G. Jones, J. S. Zabinski, Z. Czigany, and L. Hultman, J. Appl. Phys. 92, 4980 (2002).

${ }^{30}$ T. Sato, A. Narazaki, Y. Kawaguchi, and H. Niino, Appl. Phys. A: Mater. Sci. Process. 79, 1477 (2004).

${ }^{31}$ S. D. Berger, D. R. Mckenzie, and P. J. Martin, Philos. Mag. Lett. 57, 285 (1988).

${ }^{32}$ J. Fink, T. Mullerheinzerling, J. Pfluger, A. Bubenzer, P. Koidl, and G. Crecelius, Solid State Commun. 47, 687 (1983).

${ }^{33}$ S. R. P. Silva and V. Stolojan, Thin Solid Films 488, 283 (2005).

${ }^{34}$ A. J. Papworth, C. J. Kiely, A. P. Burden, S. R. P. Silva, and G. A. J. Amaratunga, Phys. Rev. B 62, 12628 (2000).

${ }^{35}$ S. E. Rodil, W. I. Milne, J. Robertson, and L. M. Brown, Appl. Phys. Lett. 77, 1458 (2000).

${ }^{36}$ A. C. Ferrari, A. Libassi, B. K. Tanner, V. Stolojan, J. Yuan, L. M. Brown, S. E. Rodil, B. Kleinsorge, and J. Robertson, Phys. Rev. B 62, 11089 (2000).

${ }^{37}$ A. C. Ferrari and J. Robertson, Phys. Rev. B 61, 14095 (2000).

${ }^{38}$ K. W. R. Gilkes, H. S. Sands, D. N. Batchelder, W. I. Milne, and J. Robertson, J. Non-Cryst. Solids 227-230, 612 (1998).

${ }^{39}$ G. Adamopoulos, J. Robertson, N. A. Morrison, and C. Godet, J. Appl. Phys. 96, 6348 (2004).

${ }^{40}$ V. I. Merkulov, J. S. Lannin, C. H. Munro, S. A. Asher, V. S. Veerasamy, and W. I. Milne, Phys. Rev. Lett. 78, 4869 (1997).

${ }^{41}$ S. Prawer, K. W. Nugent, Y. Lifshitz, G. D. Lempert, E. Grossman, J. Kulik, I. Avigal, and R. Kalish, Diamond Relat. Mater. 5, 433 (1996).

${ }^{42}$ M. Yoshikawa, Mater. Sci. Forum 52-53, 365 (1090).

${ }^{43}$ J. Tauc, R. Grigorov, and A. Vancu, Phys. Status Solidi 15, 627 (1966).

${ }^{44}$ G. E. Jellison and F. A. Modine, Appl. Phys. Lett. 69, 371 (1996).

${ }^{45}$ S. Xu, B. K. Tay, H. S. Tan, L. Zhong, Y. Q. Tu, S. R. P. Silva, and W. I. Milne, J. Appl. Phys. 79, 7234 (1996).

${ }^{46}$ G. M. Fuge, M. N. R. Ashfold, and S. J. Henley, J. Appl. Phys. 99, 014309 (2006).

${ }^{47}$ A. Chambers, Mordan Vacuum Physics (CRC, Boca Raton, FL, 2005).

${ }^{48}$ F. Claeyssens, R. J. Lade, K. N. Rosser, and M. N. R. Ashfold, J. Appl. Phys. 89, 697 (2001).

${ }^{49}$ A. C. Ferrari, S. E. Rodil, and J. Robertson, Phys. Rev. B 67, 155306 (2003).

${ }^{50}$ S. E. Rodil, S. Muhl, S. Maca, and A. C. Ferrari, Thin Solid Films 433, 119 (2003)

${ }^{51}$ J. Schwan, S. Ulrich, T. Theel, H. Roth, H. Ehrhardt, P. Becker, and S. R. P. Silva, J. Appl. Phys. 82, 6024 (1997).

${ }^{52}$ G. Lazar, K. Zellama, M. Clin, and C. Godet, Appl. Phys. Lett. 85, 6176 (2004).

${ }^{53}$ Y. Miyajima, J. M. Shannon, S. J. Henley, V. Stolojan, D. C. Cox, and S. R. P. Silva, Thin Solid Films 516, 257 (2007).

${ }^{54}$ T. Schwarz-Selinger, A. von Keudell, and W. Jacob, J. Appl. Phys. 86, 3988 (1999).

${ }^{55}$ R. C. Barklie, Diamond Relat. Mater. 10, 174 (2001).

${ }^{56}$ M. A. Monclus, D. C. Cameron, A. K. M. S. Chowdhury, R. Barkley, and M. Collins, Thin Solid Films 355-356, 79 (1999).

${ }^{57}$ F. J. Clough, W. I. Milne, B. Kleinsorge, J. Robertson, G. A. J. Amaratunga, and B. N. Roy, Electron. Lett. 32, 498 (1996).

${ }^{58}$ Y. Hayashi, N. Kamada, T. Soga, and T. Jimbo, Diamond Relat. Mater. 15, 1015 (2006). 